\title{
ON THE ABLUE OF THE SCALE PARAMETER OF THE GENERALIZED PARETO DISTRIBUTION
}

\author{
SMILEY W. CHENG AND C. H. CHOU
}

\begin{abstract}
We study the asymptotic best linear unbiased estimation of the scale parameter of the generalized Pareto distribution (GPD) with the probability density function (p.d.f.)

$$
f(x)= \begin{cases}\sigma^{-1}(1-r x / \sigma)^{1 / r-1}, & r \neq 0 \\ \sigma^{-1} \exp (-x / \sigma), & r=0 .\end{cases}
$$

In Cheng and Chou (2000), the best linear unbiased estimation of the scale parameter was discussed for finite samples. We study the large sample size cases here. Results of some chosen cases are tabulated.
\end{abstract}

\section{Introduction}

We consider the random variable $X$ having the generalized Pareto distribution (GPD) with a probability density function (p.d.f.)

$$
f(x)= \begin{cases}\sigma^{-1}(1-r x / \sigma)^{1 / r-1}, & r \neq 0 \\ \sigma^{-1} \exp (-x / \sigma), & r=0 .\end{cases}
$$

where $\sigma$ and $r$ are the scale and shape parameters respectively. The range of $x$ is $0 \leq x<\infty$ for $r \leq 0$ and $0 \leq x \leq \sigma / r$ for $r>0$.

Clearly, the standardized random variable $U=X / \sigma$ has the p.d.f.

$$
f(u)= \begin{cases}(1-r u)^{1 / r-1}, & r \neq 0 \\ \exp (-u), & r=0 .\end{cases}
$$

The range of $u$ is $0 \leq u \leq \infty$ for $r \leq 0$ and $0 \leq u \leq 1 / r$ for $r>0$.

The Pareto distribution was first proposed by Pareto (1897). The generalized Pareto distribution was introduced by Pickands (1975). Maximum likelihood estimation of generalized Pareto distribution was discussed by Davison (1984) and Smith (1984, 1985). Hosking and Wallis (1987) derived estimators of parameters and quantiles by the method

Received April 12, 2000; revised May 19, 2000.

2000 Mathematics Subject Classification. 62F99.

Key words and phrases. order statistics, sample quantiles, linear estimates, type-II censored sample. 
of moments and the method of probability weighted moments. They restricted attention to the case $-1 / 2<r<1 / 2$, for both practical and theoretical reasons. Also, they pointed out a close connection between generalized Pareto and generalized extreme-value distributions (GEV) with equal value for their shape parameters. As Hosking, Wallis, and Wood (1985) remarked, applications of the GEV distributions, particularly in hydrology usually involve the case $-1 / 2<r<1 / 2$. Chan and Cheng (1973) dealt with the asymptotically best linear unbiased estimate of the scale parameter of the Pareto distribution.

For $r<0$ the distribution has a heavy Pareto-type upper tail. The case $r=0$ is the exponential distribution for which many statistical techniques are available. When $r>0$ the distribution has an upper endpoint at $\sigma / r$. For $r=0.5$ and $r=1$ the distribution is triangular and uniform respectively.

The applications of the GDP include the use in the analysis of extreme events, in the modeling of large insurance claims, and as a failure-time distribution in reliability studies.

Consider a sample of $n$ order statistics

$$
X_{(1)}<X_{(2)}<\cdots<X_{(n)}
$$

from a continuous distribution with the p.d.f. $g[(x-\mu) / \sigma] / \sigma$, where $\mu$ and $\sigma$ are the location and scale parameters respectively.

Lloyed (1952) obtained the generalized least-square estimators of the location and scale parameters using order statistics. Cheng and Chou (2000) found the best linear unbiased estimators (BLUE's) of the parameters based on $k(\leq n)$ order statistics selected from (1.1).

Let

$$
X^{*}=\left(X_{\left(n_{1}\right)}<X_{\left(n_{2}\right)}<\cdots<X_{\left(n_{k}\right)}\right), \quad 1<n_{1}<\cdots<n_{k}<n .
$$

In estimating a parameter by a linear combination of $k(<n)$ chosen order statistics from a sample, the set of $k$ order statistics which gives the minimum variance among all possible choices of $k$ order statistics is the preferred one. However when $n$ or $k$ is large, the procedure for finding the optimum ranks is time-consuming, even with the use of a computer. Asymptotic theory is then useful for the approximation of such optimum ranks.

Ogawa (1951) proposed the asymptotically best linear unbiased estimate (ABLUE) of the location parameter $\mu$ and/or the scale parameter $\sigma$ of the distribution $g(\cdot)$ and found the optimum ranks of the ABLUE for the parameters of the normal distribution. Chan and Cheng (1973) derived the ABLUE of $\mu$ and that of $\sigma$ of the Pareto distribution.

First, we define the following terms:

Definition 1.1. A set of $k$ fixed values $\left\{\lambda_{i}\right\}=\left\{\lambda_{1}, \ldots, \lambda_{k}\right\}$ is called a spacing if it satisfies the following relation:

$$
0<\lambda_{1}<\lambda_{2}<\cdots<\lambda_{k}<1 .
$$


Definition 1.2. Let $X_{(1)}<X_{(2)}<\cdots<X_{(n)}$ be a complete sample, if only the order statistics

$$
X_{(1)}<X_{(2)}<\cdots<X_{([n \beta]+1)}, \quad 0<\beta<1,
$$

where [a] is a Gauss' symbol representing the integer part of $a$, are available in which the largest $100(1-\beta) \%$ of the order statistics in (1.1) are missing, the sample is called a right censored sample.

Definition 1.3. If only the order statistics

$$
X_{([n \alpha]+1)}<X_{[n \alpha]+2)}<\cdots<X_{(n)}, \quad 0<\alpha<1
$$

are available in which the smallest $100 \alpha \%$ of the order statistics in (1.1) are missing, the sample is called a left censored sample.

Either of the two above cases is called a singly censored sample.

Definition 1.4. If only the order statistics

$$
X_{([n \alpha]+1)}<\cdots<X_{([n \beta]+1)}, \quad 0<\alpha, \beta<1
$$

are available in which the smallest $100 \alpha \%$ and the largest $100(1-\beta) \%$ of the order statistics in (1.1) are missing, the sample is called a doubly censored sample.

\section{Definition 1.5.}

$$
\begin{aligned}
I^{*}(a, b) & =\left\{\left(\lambda_{1}, \ldots, \lambda_{k}\right): 0 \leq a \leq \lambda_{1} \leq \cdots \leq \lambda_{k} \leq b \leq 1\right\} \\
I^{*}(0,1) & =\left\{\left(\lambda_{1}, \ldots, \lambda_{k}\right): 0<\lambda_{1}<\cdots<\lambda_{k}<1\right\} \\
I(0, \beta) & =\left\{\left(\lambda_{1}, \ldots, \lambda_{k}\right): 0 \leq \lambda_{1} \leq \cdots \leq \lambda_{k} \leq \beta<1\right\} \\
I(\alpha, 1) & =\left\{\left(\lambda_{1}, \ldots, \lambda_{k}\right): 0<\alpha \leq \lambda_{1}<\cdots<\lambda_{k}<1\right\} \\
I(\alpha, \beta) & =\left\{\left(\lambda_{1}, \ldots, \lambda_{k}\right): 0<\alpha \leq \lambda_{1}<\cdots<\lambda_{k} \leq \beta<1\right\} .
\end{aligned}
$$

By applying the Gauss-Markov theorem, Ogawa (1951) obtained the ABLUE $\mu^{*}$ of $\mu$ and/or the ABLUE $\sigma^{*}$ of $\sigma$, which is a linear combination of the sample quantiles

$$
X_{\left(n_{1}\right)}<X_{\left(n_{2}\right)}<\cdots<X_{\left(n_{k}\right)}, \quad n_{i}=\left[n \lambda_{i}\right]+1
$$

When $\mu$ is known, the ABLUE $\sigma^{*}$ of $\sigma$ is

$$
\begin{aligned}
\sigma^{*} & =\sum_{i=1}^{k} b_{i} X_{\left(n_{i}\right)}-\mu \frac{K_{3}}{K_{2}} \\
\operatorname{Var}\left(\sigma^{*}\right) & =\frac{\sigma^{2}}{n K_{2}},
\end{aligned}
$$


where $K_{2}=\sum_{i=1}^{k} u_{i} K_{2 i}, K_{3}=\sum_{i=1}^{k} K_{2 i}$ and $b_{i}=K_{2 i} / K_{2}$, and

$$
\begin{aligned}
K_{2 i} & =\left\{\left(\frac{u_{i} f_{i}-u_{i-1} f_{i-1}}{\lambda_{i}-\lambda_{i-1}}\right)-\left(\frac{u_{i+1} f_{i+1}-u_{i} f_{i}}{\lambda_{i+1}-\lambda_{i}}\right)\right\} \cdot f_{i}, \\
\text { set } \lambda_{0} & =0, \lambda_{k+1}=1, f_{0}=f_{k+1}=u_{0} f_{0}=u_{k+1} f_{k+1}=0, f_{i}=f\left(u_{i}\right), i=1, \ldots, k .
\end{aligned}
$$

$K_{2}$ can easily be reduced to a simple form:

$$
K_{2}=\sum_{i=1}^{k+1} \frac{u_{i} f_{i}-u_{i-1} f_{i-1}}{\lambda_{i}-\lambda_{i-1}}
$$

\section{The ABLUE}

We now deal with the ABLUE of the scale parameter, when the shape parameter is known, of the generalized Pareto distribution based on order statistics selected from a complete, singly or doubly censored sample when the sample size is large. We apply a unified method proposed by Cheng (1975) to derive the estimate and the optimum spacing.

We first state basic lemmas and theorems which will lead to the optimum spacing algorithms and tables used to facilitate the computations.

Firstly we will deal with the complete sample, and some tables are presented. Then we will deal with left, right, and doubly censored samples and tables are presented. Finally we obtain the solution of a special case where the shape parameter $r=-1$ and give some remarks.

Assume that the value of the shape parameter $r$ is known and the scale parameter $\sigma$ is to be estimated.

The $k$ order statistics

$$
X_{\left(\left[n \lambda_{1}\right]+1\right)}<X_{\left.\left(n \lambda_{2}\right]+1\right)}<\cdots<X_{\left(\left[n \lambda_{k}\right]+1\right)}
$$

in (1.1), where $\left[n \lambda_{i}\right]$ represents the largest integer not exceeding $n \lambda_{i}$, are called sample quantiles. Ogawa's (1951) asymptotically best linear unbiased estimate of $\sigma$, when $r$ is known $(r \neq 0)$, is

$$
\begin{aligned}
\sigma^{*} & =\sum_{i=1}^{k} b_{i} X_{\left(\left[n \lambda_{i}\right]+1\right)} \\
b_{i} & =\frac{f_{i}}{K_{2}}\left[\frac{u_{i} f_{i}-u_{i-1} f_{i-1}}{\lambda_{i}-\lambda_{i-1}}-\frac{u_{i+1} f_{i+1}-u_{i} f_{i}}{\lambda_{i+1}-\lambda_{i}}\right], \quad 1=1, \ldots, k \\
K_{2} & =\sum_{i=1}^{k+1}\left(u_{i} f_{i}-u_{i-1} f_{i-1}\right)^{2} /\left(\lambda_{i}-\lambda_{i-1}\right),
\end{aligned}
$$


where

$$
\begin{aligned}
u_{i} & =F^{-1}\left(\lambda_{i}\right)=\frac{1-\left(1-\lambda_{i}\right)^{r}}{r}, f_{i}=f\left(u_{i}\right)=\left(1-\lambda_{i}\right)^{1-r}, \\
f_{0} u_{0} & =f_{k+1} u_{k+1}=0, \lambda_{0}=0, \lambda_{k+1}=1 .
\end{aligned}
$$

The asymptotic variance of $\sigma^{*}$ is

$$
\operatorname{Var}\left(\sigma^{*}\right)=\frac{\sigma^{2}}{K_{2} n}
$$

The value of $K_{2}$ in (3.4) increases with the number of $\lambda_{i}$ 's. We set $g_{i}=u_{i} f_{i}$. So, as $k \rightarrow \infty$ and $\max \left|\lambda_{i}-\lambda_{i-1}\right|$ tends to zero.

$$
\begin{aligned}
\lim _{k \rightarrow \infty} K_{2}\left(\lambda_{1}, \ldots, \lambda_{k}\right) & =\lim _{k \rightarrow \infty} \sum_{i=1}^{k}\left[\frac{g_{i}-g_{i-1}}{\lambda_{i}-\lambda_{i-1}}\right]^{2}\left(\lambda_{i}-\lambda_{i-1}\right) \\
& =\int_{0}^{1}\left(\frac{d g}{d \lambda}\right)^{2} d \lambda=\int_{0}^{1}\left(1+\frac{u f^{\prime}}{f}\right)^{2} d F=\int_{0}^{1}\left(\frac{u f^{\prime}}{f}\right)^{2} d F-1=\frac{1}{1-2 r} .
\end{aligned}
$$

Thus, the amount of information of the original sample with respect to $\sigma$ is $I(\sigma)=$ $\frac{n}{(1-2 r) \sigma^{2}}$. So the Crámer-Rao lower bound for the regular unbiased estimator of $\sigma$ is $\frac{(1-2 r) \sigma^{2}}{n}$, then the asymptotic relative efficiency of the estimator $\sigma^{*}$ is

$$
\operatorname{ARE}\left(\sigma^{*}\right)=(1-2 r) \frac{\sigma^{2}}{n} \cdot \frac{n K_{2}}{\sigma^{2}}=(1-2 r) \cdot K_{2} .
$$

In order to determine the $k$ optimum quantiles for the ABLUE of $\sigma$, we have to minimize $\operatorname{VAR}\left(\sigma^{*}\right)$ in (3.5) with respect to the standardized random variables. Equivalently, we maximize $K_{2}$ defined in $I^{*}(0,1)$ with respect to $\lambda_{i}$. We shall need the following Lemmas in the sequel. The proof for the Lemma 3.1 and 3.2 can be found in Chan and Kabir (1969), and Cheng (1975).

Lemma 3.1. $K_{2}$ in $(3.4)$ defined on $I^{*}(0,1)$ attains its maximum at an interior point of $I^{*}(0,1)$.

Lemma 3.2. A point which maximizes $K_{2}$ in (3.4) defined on $I(0,1)$ must satisfy the equations

$$
\left.G\left(\lambda_{i+1}, \lambda_{i}, \lambda_{i-1}\right)=\frac{\left(g_{i+1}-g_{i}\right)}{\lambda_{i+1}-\lambda_{i}}+\frac{\left(g_{i}-g_{i-1}\right.}{\lambda_{i}-\lambda_{i-1}}\right)-2 \cdot g_{i}^{\prime}=0, \quad i=1,2, \ldots, k,
$$

where $0=\lambda_{0}<\lambda_{i}<\cdots<\lambda_{k}<\lambda_{k+1}=1$, and $g_{i}^{\prime}=g^{\prime}\left(\lambda_{i}\right)=d g\left(\lambda_{i}\right) / d \lambda_{i}$.

Lemma 3.3. Let

$$
\begin{aligned}
& H_{1}(y)=\frac{1}{2}+\frac{y^{\frac{1}{r}-1}-1}{2(r-1)\left(y^{\frac{1}{r}}-1\right)}, \\
& H_{2}(y)=-\frac{1}{2}-\frac{y^{\frac{1}{r}}-y}{2(r-1)\left(y^{\frac{1}{r}}-1\right)}, \\
& G_{1}(y)=-H_{2}(y), G_{2}(y)=-H_{1}(y), \quad 0 \leq a \leq y \leq b \leq 1, r \neq 1,
\end{aligned}
$$


then for any fixed $y^{\prime} \in[a, b]$, there exists a unique $y^{\prime \prime} \in[a, b]$ such that

$$
\begin{array}{ll}
H_{1}\left(y^{\prime}\right)=H_{2}\left(y^{\prime \prime}\right), & 1 \leq r \leq 0.5, \\
G_{2}\left(y^{\prime}\right)=G_{1}\left(y^{\prime \prime}\right), & r<0 .
\end{array}
$$

Proof. First, we consider the case that $r \in(0,0.5)$. We have $H_{1}(1)=H_{2}(1)=0$ and the first derivative of $H_{1}(y)$ and $H_{2}(y)$ are given by

$$
H_{1}^{\prime}(y)=\frac{y^{(1 / r)-2} \cdot f_{1}(y)}{2 \cdot(r-1) \cdot\left(y^{1 / r}-1\right)^{2}},
$$

where $f_{1}(y)=r\left(1-y^{1 / r}\right)+(y-1)$,

$$
H_{2}^{\prime}(y)=\frac{f_{2}(y)}{2 \cdot(r-1) \cdot\left(y^{1 / r}-1\right)^{2}}
$$

where $f_{2}(y)=r\left(1-y^{1 / r}\right)+(y-1)$.

Since $f_{1}^{\prime}(y)=1-y^{(1 / r)-1}$ and $f_{2}^{\prime}(y)=(1 / r) \cdot(1-r) \cdot(y-1) \cdot y^{(1 / r)-2}$, for $r \in(0,0.5)$, we have $f_{1}(y)<0$ and $f_{2}(y)<0$. It follows that $H_{1}^{\prime}(y)>0$ and $H_{2}^{\prime}(y)>0$. This implies that both $H_{1}(y)$ and $H_{2}(y)$ are increasing functions of $y$. Moreover, $H_{1}(y)<0$ and $H_{2}(y)<0$. Define

$$
\begin{aligned}
H(y) & =H_{1}(y)-H_{2}(y) \\
& =1+\frac{(y-1) \cdot\left(y^{1 / r}-y\right)}{2 \cdot(r-1) \cdot y \cdot\left(y^{1 / r}-1\right)}, \quad 0<y<1 .
\end{aligned}
$$

Then

$$
H^{\prime}(y)=\frac{g_{1}(y)}{2 \cdot(r-1) \cdot\left(y^{1 / r}-1\right)^{2}}
$$

where

$$
\begin{aligned}
& g_{1}(y)=y^{\frac{1}{r}-2}\left(1-\frac{1}{r}\right)-y^{\frac{1}{r}-2}+1+\left(\frac{1}{r}-1\right) y^{\frac{1}{r}}, \text { and } \\
& g_{1}^{\prime}(y)=\left(\frac{1}{r}-1\right) y^{\frac{1}{r}-3} g_{2}(y) \\
& g_{2}(y)=\frac{1}{r} y^{2}-2 y^{\frac{1}{r}}-\frac{1}{r}+2 \\
& g_{2}^{\prime}(y)=\frac{1}{r} y\left(1-y^{\frac{1}{r}-2}\right), g_{1}(1)=g_{2}(1)=0 .
\end{aligned}
$$

Since $0<r<0.5$, we have $g_{2}^{\prime}(y)>0$ and $g_{2}(1)=0$, and therefore $g_{2}(y)<0$. It follows that $g_{1}^{\prime}(y)<0$ and $g_{1}(1)=0$. So $g_{1}(y)>0$, and $H^{\prime}(y)<0$ and $H(1)=0$. Hence, $H(y)>0$. We have $H_{1}(y)>H_{2}(y), 0<y<1$. Moreover, $H_{1}(0)=\lim _{y \rightarrow 0} H_{1}(y)=$ $r /[2 \cdot(r-1)]$, and $H_{2}(0)=-1 / 2$. Thus, we obtain a unique $y^{\prime \prime} \in\left[y^{\prime}, 1\right]$ such that $H_{1}\left(y^{\prime}\right)=H_{2}\left(y^{\prime \prime}\right)$. We have proved this Lemma for the shape parameter $r \in(0,0.5)$. 
Secondly, when $r<0$, we also have $G_{1}(1)=G_{2}(1)=0$. The first derivative of $G_{1}(y)$ and $G_{2}(y)$ are given by $G_{1}^{\prime}(y)=-H_{2}^{\prime}(y)$ and $G_{2}^{\prime}(y)=-H_{1}^{\prime}(y)$. For the shape parameter $r<0, f_{2}^{\prime}(y)>0$ and $f_{2}(1)=0$, it implies $f_{2}(y)<0$. It follows that $G_{1}^{\prime}(y)<0$ and $G_{1}(1)=0$, then $G_{1}(y)>0$. Similarly, $f_{1}^{\prime}(y)<0$ and $f_{1}(1)=0$, it implies $f_{1}(y)>0$. Hence, $G_{2}^{\prime}(y)<0$, and $G_{2}(1)=0$, so $G_{2}(y)>0$. Thus, $G_{1}(y)$ and $G_{2}(y)$ are decreasing functions of $y$ for $y \in(0,1)$, and $G_{1}(y)>0$ and $G_{2}(y)>0$. The function $G(y)\left[=G_{1}(y)-G_{2}(y)\right]$ is equal to $H(y)$ defined in (3.8). When $r<0, g_{2}^{\prime}(y)>0$ and $g_{2}(1)=0$. It implies that $g_{2}(y)<0$. It follows that $g_{1}^{\prime}(y)>0$ and $g_{1}(1)=0$. Therefore, $g_{1}(y)<0$. It follows that $H^{\prime}(y)>0$ and $H(1)=0$. It implies that $H(y)<0$, thus, $G_{1}(y)<G_{2}(y), 0<y<1$. Moreover, $G_{1}(0)=r /[2 \cdot(r-1)]$ and $G_{2}(0)=\infty$. This completes the proof.

Theorem 3.1. The system of equations (3.7) has a unique solution $\left\{\lambda_{i}^{c}\right\}=$ $\left\{\lambda_{i}^{c}, \cdots, \lambda_{k}^{c}\right\}$ in $I(0,1)$ which gives the maximum of $K_{2}$ in $(3.4)$ defined on $I(0,1)$.

Proof. Let $y_{i}=\frac{t_{i+1}}{t_{i}}=\left(\frac{1-\lambda_{i+1}}{1-\lambda_{i}}\right)^{r}, i=0,1, \ldots, k$. Clearly, $0 \leq y_{i} \leq 1$ when $0<r \leq 0.5$, and

$$
\frac{g_{i+1}-g_{i}}{\lambda_{i+1}-\lambda_{i}}=\frac{1}{r} \cdot\left\{\frac{-1}{t_{i}}\left(\frac{1-\left[t_{i+1} / t_{i}\right]^{1 r-1}}{1-\left[t_{i+1} / t_{i}\right]^{1 / r}}\right)+1\right\} .
$$

Thus, (3.7) reduces to

$$
\frac{1}{r} \cdot\left\{\frac{-1}{t_{i}}\left(\frac{1-y_{i}^{1 / r-1}}{1-y_{i}^{1 / r}}\right)+1\right\}+\frac{1}{r} \cdot\left\{\frac{-1}{t_{i-1}}\left(\frac{1-y_{i-1}^{1 / r-1}}{1-y_{i-1}^{1 / r}}+1\right\}-\frac{2}{r} \cdot\left(\frac{r-1}{t_{i}}+1\right)=0, \quad i=1, \ldots, k\right.
$$

which can be written as

$$
H_{1}\left(y_{i}\right)=H_{2}\left(y_{i-1}\right), \quad i=1, \ldots, k,
$$

where the functions $H_{1}(y)$ and $H_{2}(y)$ are as in Lemma 3.3. Since $y_{k}=0$, by Lemma $3.2, y_{k-1}$ is uniquely determined by the equation $H_{2}\left(y_{k-1}\right)=H_{1}\left(y_{k}\right)=\frac{r}{2(r-1)}$ when $0<r<1 / 2$. Since $y_{k-1}$ is now known, $y_{k-2}$ is uniquely determined by the equation $H_{2}\left(y_{k-2}\right)=H_{1}\left(y_{k-1}\right)$. Continuing this process, we can obtain for any $k$, the unique set of numbers denoted by $\left\{y_{k-1}, y_{k-2}, \ldots, y_{0}\right\}$. Consequently, $\left\{\lambda_{i}\right\}$ are uniquely determined by

$$
y_{0} y_{1} \cdots y_{i-1}=\left(1-\lambda_{i}\right)^{r}, \quad \text { i.e. } \lambda_{i}=1-\left(y_{0} y_{1} \cdots y_{i-1}\right)^{1 / r}, \quad i=1, \ldots, k .
$$

This solution gives the maximum of $K_{2}$ in $(3.4)$ defined on $I(0,1)$ followed from Lemma 3.1 and Lemma 3.2. Thus this completes the proof for the case $r \in(0,0.5)$.

For $r<0, y_{i}>1$, let $z_{i}=\frac{1}{y_{i}}=\left(\frac{1-\lambda_{i}}{1-\lambda_{i+1}}\right)^{r}<1$.

Thus, equation (3.7) can be written as

$$
G_{2}\left(z_{i}\right)=G_{1}\left(z_{i-1}\right), \quad i=1, \ldots, k,
$$

where the functions $G_{1}(z)$ and $G_{2}(z)$ are as in Lemma 3.3. Since $z_{k}=0$, by Lemma 3.2, $z_{k-1}$ is uniquely determined by the equation $G_{2}\left(z_{k-1}\right)=G_{1}\left(z_{k}\right)=r /[2(r-1)], r<0$. 
Continuing this process, we can obtain for any $k$ the unique set of numbers denoted by $\left\{z_{k-1}, z_{k-2}, \ldots, z_{0}\right\}$. Consequently, $\left\{\lambda_{i}\right\}$ are uniquely determined by $z_{0} z_{1} \cdots z_{i-1}=$ $\left(1-\lambda_{i}\right)^{-r}$, i.e. $\lambda_{i}=1-\left(z_{0} z_{1} \cdots z_{i-1}\right)^{-1 / r}, i=1 \ldots, k$. This solution gives the maximum of $K_{2}$ in (3.4) defined on $I(0,1)$ followed from Lemma 3.1 and Lemma 3.2. Thus, this proves the case for $r<0$.

Table 1 lists the optimum spacing, the coefficients, the ARE of the ABLUE $\sigma^{*}$ for a GPD of $r=0.2$ from a complete sample.

Table 1. The optimum spacing, the coefficients and the ARE of the ABLUE $\sigma^{*}$ based on $k=1(1) 6$ sample quantiles from a complete sample for GPD $(r=0.2)$

\begin{tabular}{|c|c|c|c|c|c|c|c|}
\hline$i$ & 1 & 2 & 3 & 4 & 5 & 6 & $\operatorname{ARE}\left(\sigma^{*}\right)$ \\
\hline$\lambda_{i}$ & 0.8888 & & & & & & \\
\hline$b_{i}$ & 0.5625 & & & & & & 0.5710 \\
\hline$\lambda_{i}$ & 0.7706 & 0.9745 & & & & & \\
\hline$b_{i}$ & 0.4278 & 0.1748 & & & & & 0.7595 \\
\hline$\lambda_{i}$ & 0.6744 & 0.9253 & 0.9917 & & & & \\
\hline$b_{i}$ & 0.3548 & 0.1959 & 0.0801 & & & & 0.8458 \\
\hline$\lambda_{i}$ & 0.5976 & 0.8690 & 0.9699 & 0.9967 & & & \\
\hline$b_{i}$ & 0.3057 & 0.1947 & 0.1075 & 0.0439 & & & 0.8926 \\
\hline$\lambda_{i}$ & 0.5357 & 0.8132 & 0.9392 & 0.9860 & 0.9984 & & \\
\hline$b_{i}$ & 0.2695 & 0.1870 & 0.1191 & 0.0658 & 0.0269 & & 0.9209 \\
\hline$\lambda_{i}$ & 0.4850 & 0.7609 & 0.9038 & 0.9687 & 0.9928 & 0.9992 & \\
\hline$b_{i}$ & 0.2413 & 0.1774 & 0.1231 & 0.0784 & 0.0433 & 0.0177 & 0.9393 \\
\hline
\end{tabular}

Example. Let $n=60, k=4, r=0.2$. It is easy to check from Table 1 . Then the ABLUE $\sigma^{*}$ of $\sigma$ is

$$
\sigma^{*}=0.3057 \cdot x_{(36)}+0.1947 \cdot x_{(53)}+0.1075 \cdot x_{(59)}+0.0439 \cdot x_{(60)}
$$

and the $\operatorname{ARE}\left(\sigma^{*}\right)=0.8926$.

Given a left censored sample defined in Definition 1.3. $\alpha$ is the proportion of censoring on the left. For a given $k$, consider the sample quantiles $X_{(n 1)}, X_{(n 2)}, \ldots, X_{(n k)}$ with $\alpha \leq \lambda_{1}<\cdots \leq \lambda_{k}<1$ and $n_{i}=\left[n \lambda_{i}\right]+1, i=1, \ldots, k$. Define $\lambda_{0}=0$ and $\lambda_{k+1}=1$. Based on $k$ sample quantiles $X_{(n 1)}, X_{(n 2)}, \ldots, X_{(n k)}$, the ABLUE of $\sigma$ and its variance are given by (3.2) and (3.5), respectively.

In order to determine the $k$ optimum quantiles for the ABLUE of $\sigma$, we have to minimize $\operatorname{VAR}\left(\sigma^{*}\right)$ in (3.5). Equivalently, we maximize $K_{2}$ in (3.4) on $I^{*}(\alpha, 1)$, and there are two possible cases (i) $\alpha \leq \lambda_{1}^{c}$ and (ii) $\alpha>\lambda_{1}^{c}$. For case (i), we take the optimum spacing $\left\{\lambda_{i}^{c}\right\}$ for the complete sample. For case (ii), we have the following theorem: 
Theorem 3.2. The system of equations

$$
G\left(\lambda_{i+1}, \lambda_{i}, \lambda_{i-1}\right)=0, \quad i=2, \ldots, k
$$

defined on the domain $\alpha=\lambda_{1}<\cdots<\lambda_{k}<1$ has a unique solution $\left\{\lambda_{i}^{1}\right\}=\left\{\lambda_{1}^{1}, \ldots, \lambda_{k}^{1}\right\}$ which is the only spacing at which $K_{2}$ defined on $\alpha \leq \lambda_{1}<\cdots<\lambda_{k} \leq 1$ attains its maximum.

Table 2 lists the optimum spacing, the coefficients, the ARE of the ABLUE $\sigma^{*}$ for a GPD of $r=0.2$ from a left censored sample with $\alpha=0.10$.

Table 2. The optimum spacing, the coefficients and the ARE of the ABLUE $\sigma^{*}$ based on $k=1(1) 6$ sample quantiles from a left censored $\alpha=0.10$ sample for GPD $(r=-1.0)$

\begin{tabular}{|c|c|c|c|c|c|c|c|}
\hline$i$ & 1 & 2 & 3 & 4 & 5 & 6 & $\operatorname{ARE}\left(\sigma^{*}\right)$ \\
\hline$\lambda_{i}$ & 0.1000 & 0.5500 & & & & & \\
\hline$b_{i}$ & 1.6364 & 0.6694 & & & & & 0.8167 \\
\hline$\lambda_{i}$ & 0.1000 & 0.4000 & 0.7000 & & & & \\
\hline$b_{i}$ & 1.0588 & 0.7059 & 0.1765 & & & & 0.9180 \\
\hline$\lambda_{i}$ & 0.1000 & 0.3250 & 0.5500 & & & & \\
\hline$b_{i}$ & 0.8283 & 0.6451 & 0.2867 & 0.0717 & & & 0.9534 \\
\hline$\lambda_{i}$ & 0.1000 & 0.2800 & 0.4600 & 0.6400 & 0.8200 & & \\
\hline$b_{i}$ & 0.7016 & 0.5773 & 0.3247 & 0.1443 & 0.0361 & & 0.9698 \\
\hline$\lambda_{i}$ & 0.1000 & 0.2500 & 0.4000 & 0.5500 & 0.7000 & 0.8500 & \\
\hline$b_{i}$ & 0.6207 & 0.5172 & 0.3310 & 0.1862 & 0.0828 & 0.0207 & 0.9787 \\
\hline
\end{tabular}

Example. Let $n=100, k=2, r=-1.0$ and censored at $\alpha=0.10$. From Table 2, we obtain the ABLUE of $\sigma$ as

$$
\sigma^{*}=1.6364 \cdot x_{(10)}+0.6694 \cdot x_{(55)},
$$

with $\operatorname{ARE}\left(\sigma^{*}\right)=81.67 \%$.

Given a right censored sample defined on Definition 1.2. $(1-\beta)$ is the proportion of censoring on the right. For a given $k$, consider the sample quantiles $X_{(n 1)}, X_{(n 2)}, \ldots$, $X_{(n k)}$ with $0<\lambda_{1}<\cdots<\lambda_{k} \leq \beta$ and $n_{i}=\left[n \lambda_{i}\right]+1, i=1, \ldots, k$. Define $\lambda_{0}=0$ and $\lambda_{k+1}=1$. Based on $k$ sample quantiles $X_{(n 1)}, X_{(n 2)}, \ldots, X_{(n k)}$, the ABLUE of $\sigma$ and its variance are given by (3.2) and (3.5), respectively.

In order to determine the $k$ optimum quantiles for the ABLUE of $\sigma$, we have to minimize $\operatorname{VAR}\left(\sigma^{*}\right)$ in (3.5). Equivalently, we maximize $K_{2}$ in $(3.4)$ on $I^{*}(0, \beta)$. By Theorem 3.1, $K_{2}$ has a unique maximum at an interior point of $I^{*}(0,1)$. Then, we have the following two cases: (i) $\lambda_{k}^{c} \leq \beta$ (ii) $\lambda_{k}^{c}>\beta$.

In the first case, we take the maximum of $K_{2}$ corresponding to $\left\{\lambda_{i}^{c}\right\}$, and the $k$ optimum quantiles for the complete sample case. 
For the second case, we prove the following theorem.

Theorem 3.3. The system of equations

$$
G\left(\lambda_{i+1}, \lambda_{i}, \lambda_{i-1}\right)=0, \quad i=1, \ldots, k-1,
$$

defined on the domain $0<\lambda_{i}<\cdots<\lambda_{k}=\beta$ has a unique solution $\left\{\lambda_{i}^{r}\right\}=\left\{\lambda_{i}^{r}, \ldots, \lambda_{i}^{r}\right\}$ which is the only spacing at which defined on $0<\lambda_{i}<\cdots<\lambda_{k} \leq \beta$ attains its maximum.

Given a doubly censored sample defined on Definition 1.4. $\alpha$ and $(1-\beta)$ are the proportion of censoring on the left and right. For a given $k$, consider the sample quantiles $X_{(n 1)}, X_{(n 2)}, \ldots, X_{(n k)}$ with $\alpha \leq \lambda_{i}<\cdots<\lambda_{k} \leq \beta$ and $n_{i}=\left[n \lambda_{i}\right]+1, i=1, \ldots, k$. Define $\lambda_{0}=0$ and $\lambda_{k+1}=1$. Based on $k$ sample quantiles $X_{(n 1)}, X_{(n 2)}, \ldots, X_{(n k)}$, the ABLUE of $\sigma$ and its variance are given by (3.2) and (3.5), respectively.

In order to determine the $k$ optimum quantiles for the ABLUE of $\sigma$, we have to minimize $\operatorname{VAR}\left(\sigma^{*}\right)$ in (3.5). Equivalently, we maximize $K_{2}$ in $(3.4)$ on $I^{*}(\alpha, \beta)$. By Theorem $3.1, K_{2}$ has a unique maximum at an interior point of $I^{*}(0,1)$. Then we have to consider the following five cases:

(1) $\alpha \leq \lambda_{1}^{c}, \beta>\lambda_{k}^{c}$

(2) $\alpha \leq \lambda_{1}^{r}, \beta>\lambda_{k}^{r}<\lambda_{k}^{c}$;

(3) $\alpha \leq \lambda_{1}^{r}, \beta>\lambda_{k}^{r}<\lambda_{k}^{c}$;

(4) $\lambda_{1}^{c}<\alpha=\lambda_{1}^{1}, \beta \geq \lambda_{k}^{1}$

(5) $\lambda_{1}^{c}<\alpha=\lambda_{1}^{1}, \beta \geq \lambda_{k}^{1}$.

In cases (1), (2) and (4) we take optimum spacing $\left\{\lambda_{i}^{c}\right\},\left\{\lambda_{i}^{r}\right\},\left\{\lambda_{i}^{1}\right\}$, respectively, which corresponds the maximum of $K_{2}$ defined on $I^{*}(\alpha, \beta)$. Thus, the optimum quantiles in cases (1), (2), (4) are the same as those in the complete, right, left censored sample, respectively.

Theorem 3.4. Let $\left(\lambda_{i}^{r}, \ldots, \lambda_{k}^{r}\right)$, with $0<\lambda_{i}^{r}<\cdots<\lambda_{k}^{r}=\beta$ be the optimum spacing for the right censoring sample with $\beta<\lambda_{k}^{c}$, and $\alpha>\lambda_{1}^{r}$. Then, $K_{2}$ in (3.4) defined on $I^{*}(\alpha, \beta)$ has a unique maximum at the point in $I(\alpha, \beta)$ which is the unique solution of the system of equations

$$
G\left(\lambda_{i+1}, \lambda_{i}, \lambda_{i-1}\right)=0, \quad i=2, \ldots, k
$$

with $\alpha=\lambda_{1}<\lambda_{2}<\cdots<\lambda_{k-1}<\lambda_{k}=\beta$.

To deal with case (5), we have the following theorem:

Theorem 3.5. Let $\left(\lambda_{i}^{1}, \ldots, \lambda_{k}^{1}\right)$, with $\alpha<\lambda_{i}^{1}<\cdots<\lambda_{k}^{1}<1$, be the optimum spacing for left censoring at $\beta<\lambda_{k}^{1}$, and $\alpha>\lambda_{1}^{c}$. Then $K_{2}$ in (3.4) defined on $I^{*}(\alpha, \beta)$ has a unique maximum at the point in $I(\alpha, \beta)$ which is the unique solution of the system of equations

$$
G\left(\lambda_{i+1}, \lambda_{i}, \lambda_{i-1}\right)=0, \quad i=2, \ldots, k-1
$$


with $\alpha=\lambda_{1}<\lambda_{2}<\cdots<\lambda_{k-1}<\lambda_{k}=\beta$.

When $r=-1.0$, the p.d.f of GPD is

$$
f(u)=\frac{1}{(1+u)^{2}}, \quad u>0 .
$$

And $g_{i}=u_{i} f_{i}=\lambda_{i}\left(1-\lambda_{i}\right), g_{i}^{\prime}=1-2 \lambda_{i}, f_{i}=\left(1-\lambda_{i}\right)^{2}$. Since $\frac{g_{i+1}-g_{i}}{\lambda_{i+1}-\lambda_{i}}=1-\lambda_{i+1}-\lambda_{i}$, then $G\left(\lambda_{i+1}, \lambda_{i}, \lambda_{i-1}\right)$ in (3.6) is reduced to

$$
\left(1-\lambda_{i+1}-\lambda_{i}\right)+\left(1-\lambda_{i}-\lambda_{i-1}\right)-2 \cdot\left(1-2 \lambda_{i}\right)=0, \quad i=1, \ldots, k .
$$

Thus, we obtain the following equation

$$
\lambda_{i+1}-\lambda_{i}=\lambda_{i}-\lambda_{i-1}, \quad i=1, \ldots, k .
$$

Since $\lambda_{0}=0$, then $\lambda_{2}=2 \cdot \lambda_{1}$. Substitute $\lambda_{2}$ into (3.10) to obtain $\lambda_{3}=3 \cdot \lambda_{1}$. Proceeding in this way, we can obtain $\lambda_{i}=i \cdot \lambda_{1}$. Since $\lambda_{k+1}=1$, it follows from $\lambda_{k+1}=(k+1) \cdot \lambda_{1}$ that $\lambda_{1}=1 /(k+1)$. Thus, we obtain

$$
\lambda_{i}=i /(k+1), \quad i=1, \ldots, k .
$$

Substitute (3.11) into (3.4) and (3.3) to obtain $K_{2}$ and $b_{i}$ as follows:

$$
\begin{aligned}
K_{2} & =\frac{k(k+2)}{3(k+1)^{2}}, \\
b_{i} & =\frac{6(k+1-i)^{2}}{k(k+1)(k+2)}, \quad i=1, \ldots, k .
\end{aligned}
$$

The $\operatorname{ARE}\left(\sigma^{*}\right)$, defined on $(3.6)$, is

$$
\operatorname{ARE}\left(\sigma^{*}\right)=\frac{k(k+2)}{(k+1)^{2}} .
$$

Table 3 lists the optimum spacing, the coefficients, the ARE of the ABLUE of $\sigma$ of a GPD from a doubly censored sample with $\alpha=0.4$ and $\beta=0.8$.

Table 3. The optimum spacing, the coefficients and the ARE of the ABLUE $\sigma^{*}$ of $\sigma$ of a GDP of $r=-0.5$ based on $k=2(1) 5$ sample quantiles from a Doubly censored sample for proportions of censoring $\alpha=0.4$ and $\beta=0.8$.

\begin{tabular}{|c|c|c|c|c|c|c|}
\hline$i$ & 1 & 2 & 3 & 4 & 5 & $\operatorname{ARE}\left(\sigma^{*}\right)$ \\
\hline$\lambda_{i}$ & 0.4000 & 0.8000 & & & & \\
\hline$b_{i}$ & 0.8574 & 0.2027 & & & & 0.8669 \\
\hline$\lambda_{i}$ & 0.4000 & 0.6176 & 0.8000 & & & \\
\hline$b_{i}$ & 0.5879 & 0.2516 & 0.1405 & & & 0.9138 \\
\hline$\lambda_{i}$ & 0.4000 & 0.5487 & 0.6827 & 0.8000 & & \\
\hline$b_{i}$ & 0.5068 & 0.2075 & 0.1298 & 0.1218 & & 0.9226 \\
\hline$\lambda_{i}$ & 0.4000 & 0.5129 & 0.6177 & 0.7137 & 0.8000 & \\
\hline$b_{i}$ & 0.4674 & 0.1719 & 0.1244 & 0.0846 & 0.1126 & 0.9257 \\
\hline
\end{tabular}


Example. Let $n=100, k=2, \alpha=0.4, \beta=0.8$. It is easy to obtain the optimum quantiles from Table 3 as $X_{(40)}, X_{(80)}$. The ABLUE of $\sigma$ is

$$
\sigma^{*}=0.8574 \cdot X_{(40)}+0.2027 \cdot X_{(80)}
$$

with $\operatorname{ARE}\left(\sigma^{*}\right)=86.69 \%$.

\section{The Algorithm}

We must use an algorithm to find the optimum spacing $\left\{\lambda_{i}\right\}$ for the ABLUE $\sigma^{*}$ of $\sigma$ based on complete sample or censored sample.

Let $y_{i}=\left[\left(1+\lambda_{i-1}\right) /\left(1-\lambda_{i}\right)\right]^{r}, i=1, \ldots, k, 0<r<1 / 2$.

Now we state the algorithm as follows:

(a) complete sample

$$
\begin{gathered}
y_{0} y_{1} \cdots y_{i-1}=\left(\frac{1-\lambda_{1}}{1-\lambda_{0}} \cdot \frac{1-\lambda_{2}}{1-\lambda_{1}} \cdots \frac{1-\lambda_{i}}{1-\lambda_{i-1}}\right)^{r}=\left(1-\lambda_{i}\right)^{r}, \\
\Rightarrow \lambda_{i}=1-\left(y_{0} y_{i} \cdots y_{i-1}\right)^{1 / r}, i=1,2, \ldots, k
\end{gathered}
$$

(b) Left censored sample at $\alpha$

$$
\begin{aligned}
y_{0} y_{1} \cdots y_{i-1} & =\left(\frac{1-\lambda_{i}}{1-\lambda_{1}}\right)^{r}=\left(\frac{1-\lambda_{i}}{1-\alpha}\right)^{r}, \\
\Rightarrow \lambda_{i} & =1-(1-\alpha) \cdot\left(y_{0} y_{1} \cdots y_{i-1}\right)^{1 / r}, \quad i=1,2, \ldots, k . \\
\lambda_{1} & =\alpha .
\end{aligned}
$$

(c) Right censored sample at $\beta$

$$
\begin{aligned}
y_{i} y_{i+1} \cdots y_{k-1} & =\left(\frac{1-\lambda_{k}}{1-\lambda_{i}}\right)^{r}=\left(\frac{1-\beta}{1-\lambda_{i}}\right)^{r} \\
\Rightarrow \lambda_{i} & =1-(1-\beta) \cdot\left(y_{i} y_{i+1} \cdots y_{k-1}\right)^{1 / r}, \quad i=1,2, \ldots, k-1, \\
\lambda_{i} & =\beta
\end{aligned}
$$

(d) Doubly censored sample at $\alpha$ and $\beta$

$$
\begin{aligned}
\lambda_{i} & =1-(1-\alpha) \cdot\left(y_{0} y_{1} \cdots y_{i-1}\right)^{1 / r} \\
\text { or } \lambda_{i} & =1-(1-\beta) \cdot\left(y_{i} y_{i+1} \cdots y_{k-1}\right)^{1 / r}, \quad i=1,2, \ldots, k-1, \\
\lambda_{1} & =\alpha, y_{k}=\beta .
\end{aligned}
$$

When $r<0$, the algorithm is the same as above except

$$
y_{i}=\left(\frac{1-\lambda_{i}}{1-\lambda_{i+1}}\right)^{r}, \quad i=1, \ldots, k .
$$


FORTRAN 77 programs were written in order to find the values of the optimum spacing, the corresponding coefficients and the ARE for the complete sample, singly censored sample and doubly censored sample cases and any values of $k$ and $r$. We listed one table for each case and the other cases can similarly be derived using the algorithm above.

All computations were rounded to the $4^{\text {th }}$ decimal place for the complete and the censored samples.

\section{Conclusion}

In this paper we can see from the tables that most of the relative efficiencies are quite high for the ABLUE of $\sigma$. From the results it is easy to obtain the ABLUE of the scale parameter $\sigma$ of the GPD for the various shape parameters. If the shape parameter $r<0$, then the ABLUE of $\sigma$ is very good. The ABLUE of $\sigma$ for the shape parameter $0<r<1 / 2$ has smaller relative efficiency than that for $r<0$.

\section{Acknowledgement}

The Authors are indebted to the referee and the Editor for their helpful comments and suggestions which have vastly improved the article. The first author's research was partially supported by a grant from Natural Sciences and Engineering Research Council, Canada.

\section{References}

[1] L. K. Chan and S. W. Cheng, On the optimum spacing for the asymptotically best linear estimate of the scale parameter of the Pareto distribution, Tamkang Journal of Mathematics, 4(1973), 9-21.

[2] L. K. Chan and A. B. M. L. Kabir, Optimum Quantiles for the linear estimation of the parameters of the extreme value distribution in complete and censored samples, Naval Research Logistics Quarterly, 16(1969), 381-404.

[3] S. W. Cheng, A unified approach to choosing optimum for the ABLE's, Journal of the American Statistical Association, 70(1975), 155-159.

[4] S. W. Cheng and C. H. Chou, On the BLUE of the scale parameter of the generalized Pareto distribution, Tamkang Journal of Mathematics, 31(2000), to appear.

[5] A. C. Davison, Modeling excesses over high thresholds, in statistical extremes and application, ed. J. Tiago de Oliveira, Dordrecht: D. Reidel (1984), 461-482.

[6] J. R. M. Hosking and J. R. Wallis, Parameter and quantile estimation for the generalized Pareto distribution, Technometrics, 29(1987), 339-349.

[7] J. R. M. Hosking, J. R. Wallis and E. F. Wool, Estimation of the generalized extremevalue distribution by the method of probability weighted moments, Technometrics, 27(1985), 251-261.

[8] E. H. Lloyd, Least-squares estimation of location and scale parameters using order statistics, Biometrics, 39(1952), 88-95. 
[9] J. Qgawa, Contributions to the theory of systematics, I. Osaka, Mathematical Journal, 3(1951), 175-213.

[10] V. Pareto, Cours d'Ecomonie Politique Rouge et Cie, Lausanne and Paris, 1987.

[11] J. Pickands, Statistical inference using extreme order statistics, The Annals of Statistics, 3(1975), 119-131.

[12] A. E. Sarhan and B. G. Greenberg, Contribution to order statistics, John Wiley and Sons, New York, 1962.

[13] R. L. Smith, Thereshold methods for sample extremes in statistical Extremes and Applications, ed. J. Tiago de Oliveira, Dordrecht: D. Reidel, (1984), 621-638.

[14] R. L. Smith, Maximum likelihood estimation in a class of nonregular cases, Biometrika, 72(1985), 67-90.

Department of Statistics, University of Manitoba, Winnipeg, Manitoba, R3T 2N2 Canada.

Cathay Assurance Company, Taipei, Taiwan, R.O.C. 